\title{
A Modified Rheological Model of Viscosities for BR-SBS Blends
}

\author{
LINE-HWA CHU, ${ }^{1}$ WEN-YEN CHIU, ${ }^{1}$ CHAO-HSUN CHEN, ${ }^{2}$ HSIENG-CHENG TSENG ${ }^{3}$ \\ ${ }^{1}$ Institute of Material Science and Engineering, National Taiwan University, Taipei, Taiwan, Republic of China \\ ${ }^{2}$ Institute of Applied Mechanics, National Taiwan University, Taipei, Taiwan, Republic of China \\ ${ }^{3}$ Department of Chemical Engineering, National Taiwan Institute of Technology, Taipei, Taiwan, Republic of China
}

Received 10 November 1997; accepted 4 June 1998

\begin{abstract}
Blends of polybutadiene (BR) and styrene-butadiene-styrene triblock copolymer (SBS) have been prepared by a two-roll mill. The morphologies of extruded samples from a capillary rheometer were observed by scanning electron microscopy (SEM). It is found that PS phase is dispersed in the BR phase. The glass transition temperature $\left(T_{g}\right)$ of the blend has been examined by using differential scanning calorimetry (DSC). From the $T_{g}$ behavior and the electron microscopy study, it is found that certain degree of miscibility between the polystyrene phase and the BR phase is observed. The rheological behavior of the blend has been investigated by a capillary rheometer. It is found that the viscosity of the blend increases with increased content of PS phase. The behavior is in accord with the expected behavior of filler effect. To predict the filler effect of PS phase on the BR-SBS blend, a modified model of Chen and Cheng is proposed to elucidate the rheological properties of the BR-SBS blends with different compositions. Chen and Cheng's micromechanical model derived in Part I of this series, which relates the macroscopic shear stress to the macroscopic shear rate of a rigid non-Newtonian suspension when the direct contribution of Brownian force is completely neglected. The agreement between the theoretical predictions and the experimental results is satisfactory. (c) 1999 John Wiley \& Sons, Inc. J Appl Polym Sci 71: 39-46, 1999
\end{abstract}

Key words: viscosity model, BR-SBS Blends

\section{INTRODUCTION}

The blend of polystyrene (PS) and polybutadiene (BR) shows 2 distinct glass transition temperatures corresponding to a PS phase and the other BR phase, respectively. ${ }^{1,2}$ This indicates that PS and BR are immiscible. ${ }^{3}$

From our previous results, ${ }^{1}$ it is found that the styrene component forms a discrete phase, the butadiene component forms a continuous phase

Correspondence to: W.-Y. Chiu.

Journal of Applied Polymer Science, Vol. 71, 39-46 (1999)

(C) 1999 John Wiley \& Sons, Inc.

CCC 0021-8995/99/010039-08 in BR-SBS blend, and the domain size of styrene phase is rather uniform and small. And the viscosity of BR-SBS blend increases with increased content of styrene phase, although the viscosity of PS at the same shear rate and temperature shows much lower value. It might be attributed to the strong interaction between styrene and butadiene due to chemical bonding, which plays a role like surfactants and will reduce the amount of strain within the discrete domain and the PS domain will behave like rigid particles. ${ }^{4}$ Therefore, the BR-SBS polyblend fluid can be regarded as a suspension fluid with PS domain as fillers and BR phase as a suspending medium. 
Many equations have been proposed to estimate the viscosity of a suspension in which rigid particles are dispersed in a Newtonian fluid. Einstein was the first to develop a theory for predicting the viscosity of a dilute suspension of rigid spheres. The Einstein equation ${ }^{5}$ is valid only for extremely low concentration suspensions with the suspending medium being a Netonian fluid. Many studies were carried out to extend the Einstein equation. Taylor ${ }^{6,7}$ developed a theoretical expression for a dilute emulsion dispersed in a Newtonian fluid. Jaffery ${ }^{8,9}$ considered the effect of the shape of the particles in the suspension and proposed a rigid ellipsoidal particle model. This model includes a parameter that depends on the geometry of ellipsoidal particles to modify the Einstein equation. At a higher concentration, some models have been proposed, such as the cell model by Simha ${ }^{8,10}$ and the Mooney equations, ${ }^{11}$ to consider the particle-particle interactions that could influence the blend viscosity. Chen and Cheng ${ }^{12}$ adopted Eshelby's eigen-strain ${ }^{13}$ and Mori-Tanaka's mean stress concept ${ }^{14}$ to estimate the viscosity of a solid-filled PEEK fluid. The proposed equation is able to describe the non-Newtonian behavior of a suspension.

In this article, we study the rheological properties of BR-SBS blends. The melt viscosities of BR-SBS blends behave as a PS domain filled in the suspending medium of BR melt. To describe the rheological properties of the blends as function of weight fraction of PS phase, the model proposed by Chen and Cheng ${ }^{12}$ is modified by replacing the power law model with the modified cross model to predict the rheological behavior of the BR-SBS blend.

\section{THEORETICAL CONSIDERATION}

Many equations have been proposed to estimate the viscosity of a suspension in which rigid particles are dispersed in a Newtonian fluid. Einstein was the first to develop a theory for predicting the viscosity of a dilute suspension of rigid spheres. ${ }^{5}$ The Einstein equation is given as

$$
\frac{\zeta^{S}}{\zeta_{0}}=1+2.5 \phi
$$

where $\zeta^{S}$ is the viscosity of suspension, $\zeta_{0}$ is the viscosity of suspending medium, and $\phi$ is the volume fraction of the filled particles.
In a previous report, Chen and Cheng ${ }^{12}$ adopted Eshelby's eigen-strain ${ }^{13}$ and Mori-Tanaka's mean stress concept ${ }^{14}$ to develop a model (equation) to estimate the viscosity of a solid-filled PEEK fluid. In the present study, this model is extended to predict the melt viscosity of BR-SBS polyblend. The details are given below.

For a suspension, Chen and Cheng ${ }^{12}$ assume that the relationship between shear stress and shear rate can be expressed by the following power law form:

$$
\bar{Q}=\mathrm{d} \dot{\gamma}^{\mathrm{n}}
$$

where $\bar{Q}$ and $\dot{\gamma}$ are the shear stress and shear rate, respectively, $d$ and $n$ are power law constants. The apparent viscosity $\left(\zeta^{S}\right)$ of the polymer fluid is defined as

$$
\zeta^{S}=\frac{\bar{Q}}{\dot{\gamma}}=\mathrm{d} \dot{\gamma}^{\mathrm{n}-1}
$$

For heterogeneous polyblends, the discrete phases disperse in the continuous phase. If the discrete phase can be regarded as rigid particles, and the continuous phase as the suspension medium, the viscosity of the continuous phase $\left(\zeta_{0}^{S}\right)$ is expressed as

$$
\zeta_{0}^{S}=\mathrm{d} \dot{\gamma}_{m}^{\mathrm{n}-1}
$$

and

$$
\dot{\gamma}_{m}=\frac{\dot{\gamma}}{W_{m}}
$$

where $\dot{\gamma}_{m}$ is the shear rate of the continuous phase, and $W_{m}$ is the weight fraction of the continuous phase. The apparent viscosity of the polymer blend $\left(\zeta^{S}\right)$ can then be derived as ${ }^{12}$

$$
\zeta^{S}=\frac{\zeta_{0}^{S}}{b W_{m}}
$$

For an incompressible fluid suspension, assuming that the density of continuous phase is close to 1.0 , and the disperse phase behaves as a rigid sphere; the parameter $b$ can be expressed as ${ }^{12}$

$$
b=\frac{1}{1+1.5 W_{f}}
$$


where $W_{f}$ is the weight fraction of the dispersed phase. Introducing eqs. (3) and (4) into eq. (5), it is shown that

$$
\zeta^{S}=\frac{\mathrm{d} \dot{\gamma}^{\mathrm{n}-1}}{b W_{m}^{n}}
$$

As $W_{f}=0$, the viscosity of the continuous phase $\left(\zeta_{0}\right)$ at certain shear rate $(\dot{\gamma})$ can be expressed as $\mathrm{d} \dot{\gamma}^{\mathrm{n}-1}$, so the viscosity ratio of the blend to the pure continuous phase fluid at same shear rate is given as

$$
\frac{\zeta^{S}}{\zeta_{0}}=\frac{1}{b W_{m}^{n}}
$$

We refer to eq. (8) as Chen's model..$^{12}$ However, it is known that the flow curve of viscosity versus shear rate exhibits Newtonian behavior at low shear rate and follows power law equation only over a limited range of shear rate for polymer fluid. In the present study, a modified pseudo cross equation instead of the power law equation is used to describe the flow curve of polymer fluid; that is, eq. (3) is replaced by eq. (9) as

$$
\zeta_{0}^{S}=\frac{D}{1+\Omega \dot{\gamma}_{m}^{a}}
$$

where $D$ is the zero shear rate viscosity of the blend. $\Omega$ and $a$ are material constants. Introducing eq. (5) into eq. (9), we obtain

$$
\zeta^{S}=\frac{D}{1+\Omega\left(\frac{\dot{\gamma}}{W_{m}}\right)^{a}} \times \frac{1}{b W_{m}}
$$

Similarly, the viscosity of the pure continuous phase fluid at shear rate $\dot{\gamma}$ can be expressed as

$$
\zeta_{0}=\frac{D}{1+\Omega \dot{\gamma}^{a}}
$$

So the viscosity ratio of the blend to the pure continuous phase fluid is iven as

$$
\frac{\zeta^{S}}{\zeta_{0}}=\frac{\frac{D}{1+\Omega\left(\frac{\gamma}{W_{m}}\right)^{a}} \frac{1}{b W_{m}}}{\frac{D}{1+\Omega \gamma^{a}}}=\frac{1+\Omega \gamma^{a}}{1+\Omega\left(\frac{\gamma}{W_{m}}\right)^{a}} \frac{1}{b W_{m}}
$$

It is noted that

$$
\lim _{\gamma \rightarrow 0} \frac{\zeta^{S}}{\zeta_{0}}=\frac{1}{b W_{m}}
$$

and

$$
\lim _{\gamma \rightarrow \infty} \frac{\zeta^{S}}{\zeta_{0}}=\frac{1}{b W_{m}^{1-a}}
$$

In comparison of eq. (13) to eq. (8), the constant 1 - $a$ is equal to the power law constant $\mathrm{n}$.

Equations (12) and (13) are our modified equations to calculate the viscosity variation of BRSBS blends at different compositions, which will compare with the Einstein equation [that is, eq. (1)] and Chen's model [that is, eq. (8)] over a wide range of shear rate.

\section{EXPERIMENTAL}

\section{Materials}

The materials used in this study were as follow: polystyrene (PS), $\bar{M}_{w}=260,000$; polybutadiene (BR), $\bar{M}_{w}=550,000$; and styrene-butadienestyrene copolymer (SBS), $\bar{M}_{w}=300,000$, with $30 \mathrm{wt} \%$ styrene and $70 \mathrm{wt} \%$ butadiene.

\section{Preparation of the Blends}

Polymer blends with weight ratios of $83 / 17,67 / 33$, 58/41, 50/50, 42/58, 33/67, and 17/83 (BR-SBS) were prepared in a 2 -roll mill with a surface temperature of $150^{\circ} \mathrm{C}$ and a mixing time of approximately $15 \mathrm{~min}$. The compositions of the polymer blends were carefully chosen so that when they are expressed in terms of the weight ratio of styrene (ST) to butadiene (BD), the corresponding weight ratios of styrene (ST) to butadiene (BD) for all the above blends become $5 / 95,10 / 90,12.5 / 78.5$, $15 / 85,17.5 / 82.5,20 / 80$, and $25 / 75$, respectively.

\section{Measurement of Viscosity}

The melt viscosities of PS, BR, and BR-SBS blends at $180,200,220^{\circ} \mathrm{C}$ were measured with a capillary rheometer. The Rabinowitsch correction was employed to obtain the true shear rate. 


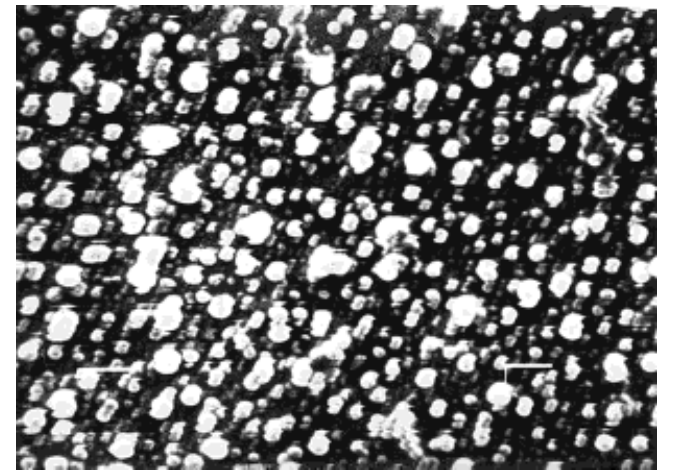

Figure 1 SEM photomicrograph $(\times 5000)$ of etched fractured surface for SBS extrudate from the capillary rheometer at $200^{\circ} \mathrm{C}$.

\section{Thermal Properties}

The glass transition temperatures $\left(T_{g}\right)$ of PS, BR, and BR-SBS blends were determined with a $\mathrm{Du}$ Pont 9900 differential scanning calorimeter over the temperature range of -140 to $160^{\circ} \mathrm{C}$ at a heating rate of $20^{\circ} \mathrm{C} / \mathrm{min}$.

\section{Morphology}

The extrudates from the capillary rheometer were freeze-fractured, and their morphologies were observed with a scanning electron microscope. The sample for scanning electron microscopy (SEM) analysis was etched with a solution that was prepared with $200 \mathrm{~mL}$ of $\mathrm{H}_{2} \mathrm{SO}_{4}, 65 \mathrm{~mL}$ of $\mathrm{H}_{3} \mathrm{PO}_{4}, 63$ $\mathrm{mL}$ of $\mathrm{H}_{2} \mathrm{O}$, and $10 \mathrm{~g}$ of $\mathrm{CrO}_{3}$. Etching was performed with samples being soaked in the solution for 3-10 min. The BR component on the surface of the sample was extracted by the solution. The samples were then rinsed with $\mathrm{H}_{2} \mathrm{O}$ and $\mathrm{C}_{2} \mathrm{H}_{5} \mathrm{OH}$ and coated with gold before SEM analysis.

\section{RESULTS AND DISCUSSION}

SBS is a triblock copolymer with styrene blocks at 2 ends and BR in the middle. There are chemical bondings between the styrene phase and butadiene phase. ${ }^{15}$ Figure 1 gives the SEM micrograph of the extrudate of SBS. The discrete phase represents the PS phase, and the continuous one represents the BR phase. The domain size of styrene phase is rather uniform and small. When BR and SBS are blended, BR will mix with the butadiene blocks of SBS. Figure 2 gives the SEM micrograph of the extrudate of the BR-SBS blend with weight ratio of 50/50 (ST to BD is 15/85). It is also seen in Figure 2 that the styrene component forms the discrete phase, and the butadiene component forms the continuous phase. The domain size of styrene phase is rather uniform and small. From our previous article, ${ }^{1}$ it is found that the discrete phase (PS phase) in the BR-SBS blend is much more uniformly dispersed than that in the PS-BR blend, and the droplet size in the BR-SBS blend is much smaller than that in the PS-BR blend. From the study of $\mathrm{Wu},{ }^{16}$ it is known that the stronger the interactions between the 2 phases are, the smaller the droplet size is. Hence, the interaction between the PS phase and BR phase in the BR-SBS blend is due mainly to the chemical bonding existing in the SBS.

The measurement of the glass transition temperature $\left(T_{g}\right)$ of a polymer blend is often used as a criterion to differentiate the degree of miscibility. ${ }^{17}$ In general, a miscible polymer blend will exhibit a single $T_{g}$ between the $T_{g}$ 's of the components. An immiscible polymer blend will exhibit 2 separate transitions that are consistent with the unblended constituents. While for partial miscible polymer systems, the $T_{g}$ 's will shift toward each other. The glass transition temperature of the BR-SBS blend is determined from differential scanning calorimetry (DSC) analysis. The glass transition temperatures of pure PS and pure BR are 98.7 and $-96.8^{\circ} \mathrm{C}$, respectively, and the $T_{g}$ 's of SBS are shifted from the values for PS and $\mathrm{BR}$ of 98.7 and $-96.8^{\circ} \mathrm{C}$ to 70.3 and $-83.8^{\circ} \mathrm{C}$. The $T_{g}$-composition relationship of BR-SBS blend is shown in Figure 3, in which the dashed line is drawn to indicate the existence of PS phase, even though it's $T_{g}$ cannot be observed due to the sensitivity of the DSC. It is seen that there

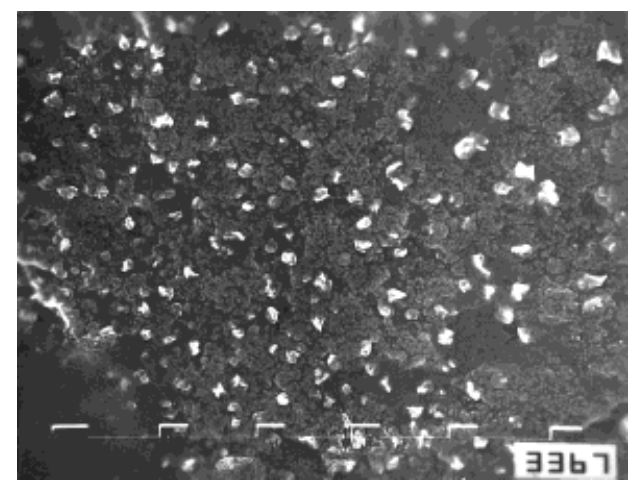

Figure 2 SEM photomicrograph $(\times 5000)$ of etched fractured surface for the BR-SBS extrudate from the capillary rheometer with a weight ratio of BR-SBS $=50 / 50($ or $\mathrm{ST} / \mathrm{BD}=15 / 85)$ at $200^{\circ} \mathrm{C}$. 


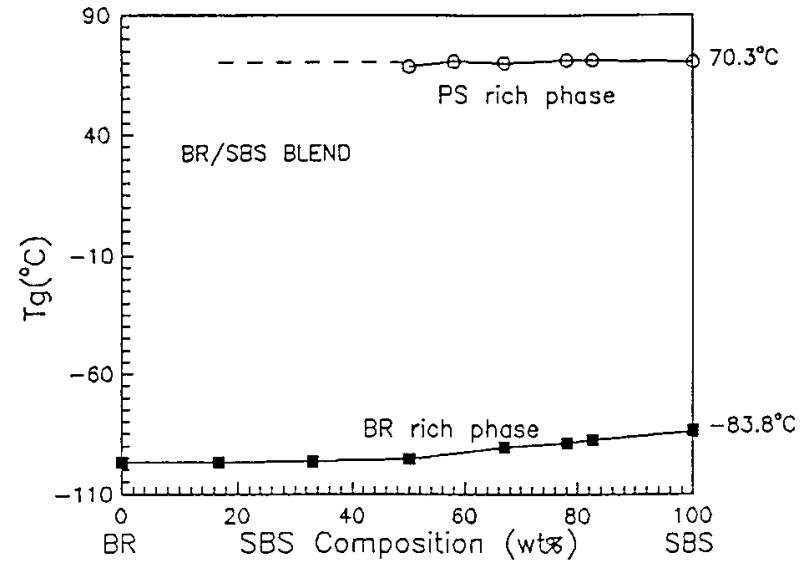

Figure $3 T_{g}(\mathrm{BR})$ and $T_{g}$ (PS) for BR-SBS blends.

are 2 glass transition temperatures for the blend, which indicates the existence of 2 phases in which $T_{g}(\mathrm{BR})$ is associated with the BR-rich phase and $T_{g}$ (PS) is associated with the PS-rich phase. The $T_{g}$ of BR-rich phase is seen to increase with increased content of SBS in the BR-SBS blend, while the $T_{g}$ of the PS-rich phase is nearly constant at $70^{\circ} \mathrm{C}$ for all BR-SBS blends. It indicates that the pure BR polymer could mix well with the BR phase in SBS but not significantly disturb the PS phase in SBS.

The viscosity curves for BR-SBS blends and pure PS at 200 and $220^{\circ} \mathrm{C}$ are shown in Figures 4 and 5 , respectively. It can be seen that as shear rate increases, the viscosity decreases, indicating

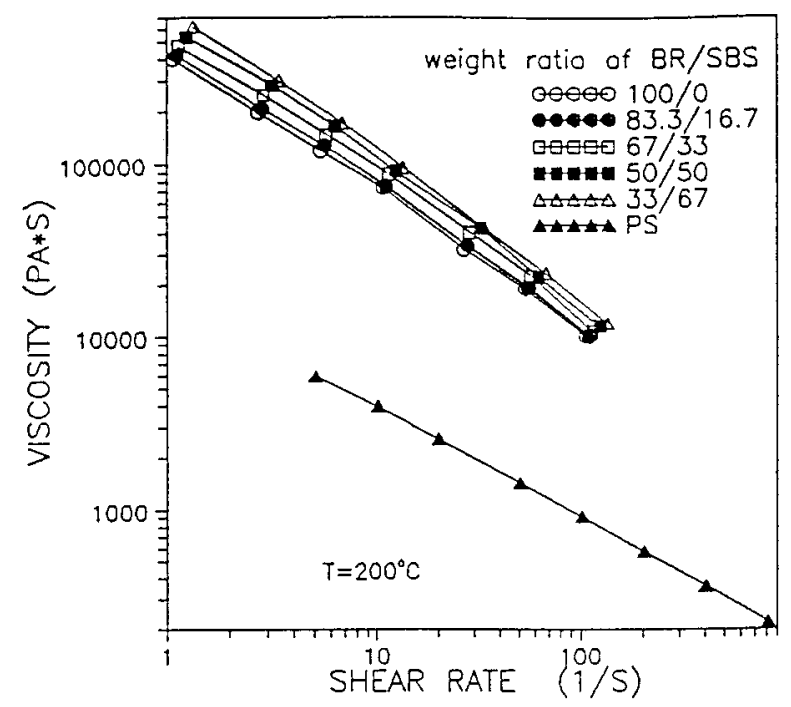

Figure 4 Viscosity versus shear rate for the BR-SBS blends and PS at $200^{\circ} \mathrm{C}$.

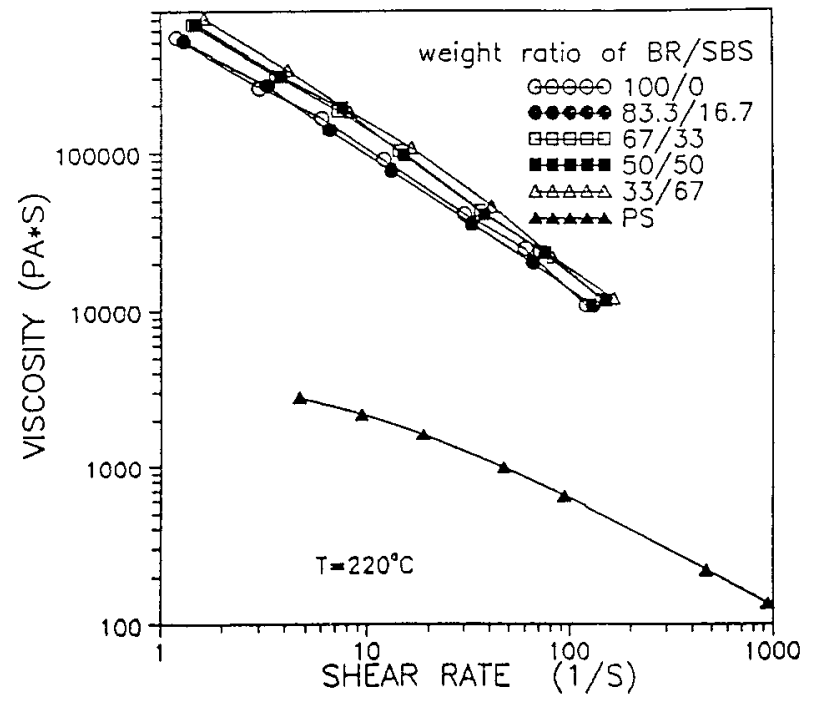

Figure 5 Viscosity versus shear rate for the BR-SBS blends and PS at $220^{\circ} \mathrm{C}$.

pseudo plastic flow behavior. We also can see that the viscosity of PS is lower than that of BR-SBS blends, but the viscosity of BR-SBS blend increases with an increase in the content of SBS. In other words, the viscosity of the BR-SBS blend increases with increased content of styrene (or PS), which indicates that PS domains act like rigid fillers in the $\mathrm{BR}$ matrix fluid. This might be due to the fact that the domain size of PS is small in the BR-SBS blend, and the interaction coming from the chemical bonding between styrene and butadiene plays a role like surfactants, which will diminish most of the strain to PS domain so that the deformation of PS domain is negligible. Oene ${ }^{4}$ stated that when a suspension of fluid droplets is subjected to flow, the stress arising in the matrix tend to deform the droplet. The stresses are continuous through the interface, thus establishing a system of velocity gradients inside the droplets, which establish streamlines inside and outside the droplets. The phenomena will form an internal circulation. The presence of surfactant may form a solid membrane around the droplets, which will reduce the amount of internal circulation within the droplets, and the droplets will behave as rigid particles.

Figure 6 shows the effect of temperature on the melt viscosities of the BR-SBS blends and PS. It is seen that for BR and all the BR-SBS blends, the viscosity increases slightly with increasing the temperature at a fixed shear rate, while the viscosity of PS decreases with an increase in the temperature at a fixed shear rate. It means that 


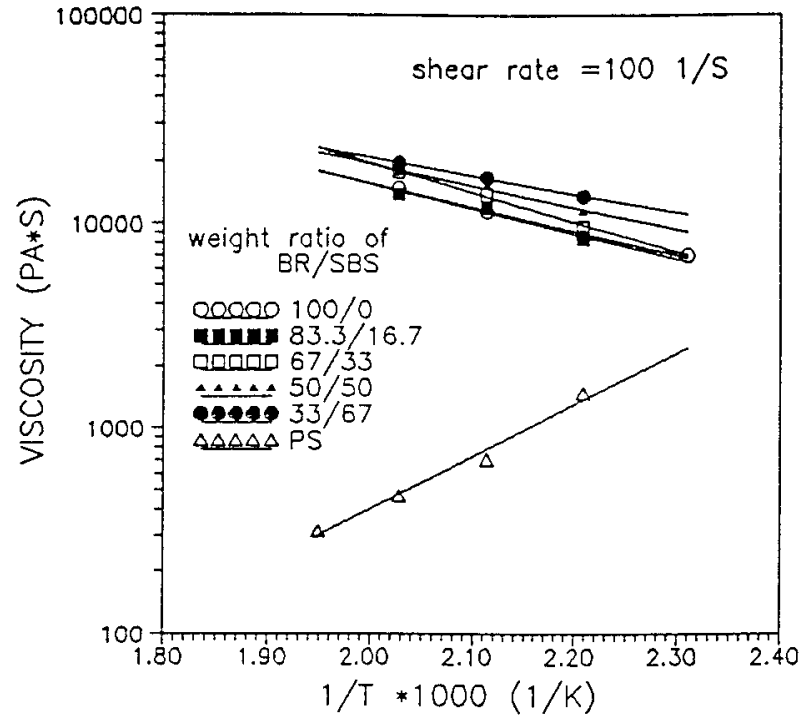

Figure 6 Temperature dependence of viscosity of the BR-SBS blends and PS at shear rate of $100 \mathrm{~S}^{-1}$.

the flow behavior of the BR-SBS blends is dominated by the BR continuous phase, and the PS dispersed phase can be treated as fillers.

In order to observe how the viscosities of the BR-SBS blends vary with the content of styrene phase, the viscosities of a series of BR-SBS blends with different compositions are measured at different shear rate and different temperatures, as shown in Figures 7-12. Meanwhile, the

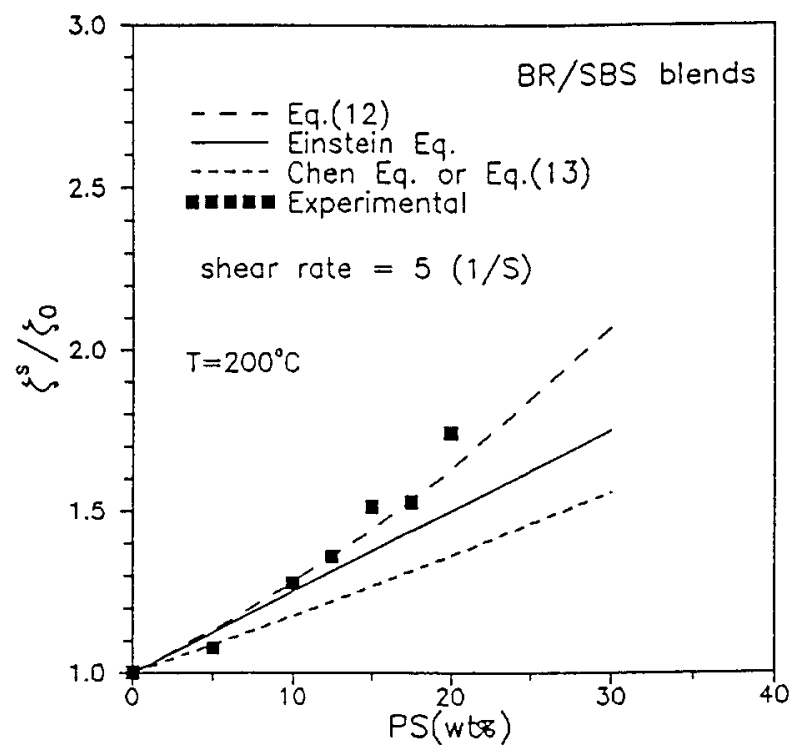

Figure 7 The relative viscosity predicted by eqs. (1), (8), (12), and (13) and experimental values measured at $200^{\circ} \mathrm{C}$ at shear rate of $5 \mathrm{~S}^{-1}$.

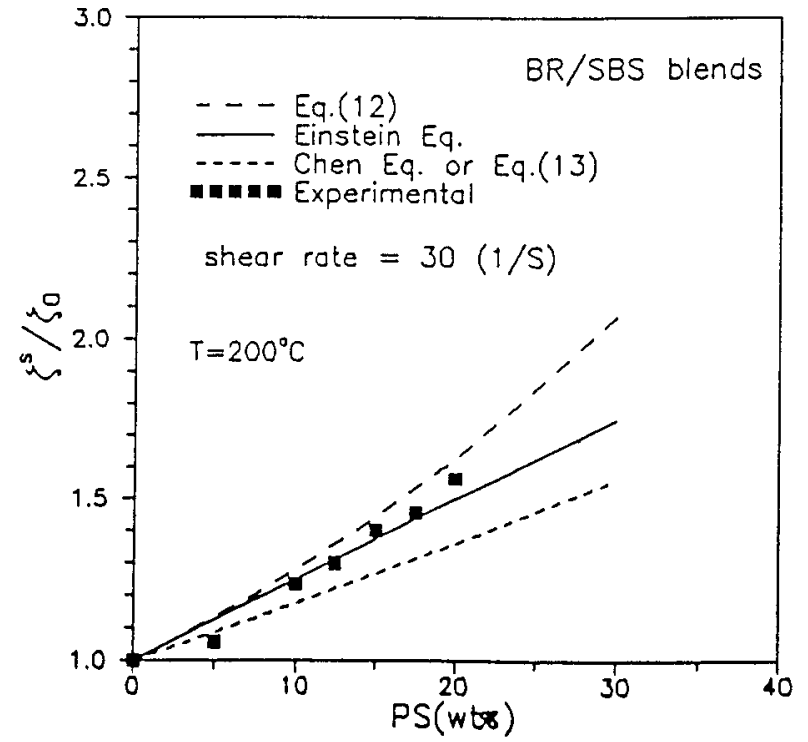

Figure 8 The relative viscosity predicted by eqs. (1), (8), (12), and (13) and experimental values measured at $200^{\circ} \mathrm{C}$ at shear rate of $30 \mathrm{~S}^{-1}$.

relative viscosity $\left(\zeta_{r}=\frac{\zeta^{S}}{\zeta_{0}}\right)$ or the viscosity ratio of the blend to the continuous phase fluid (BR) as function of the weight fraction of disperse phase (PS) can be predicted from the equation of Einstein [eq. (1)], the equation of Chen [eq. (8)], and our modified model [eqs. (12) and (13)]. To use eq.

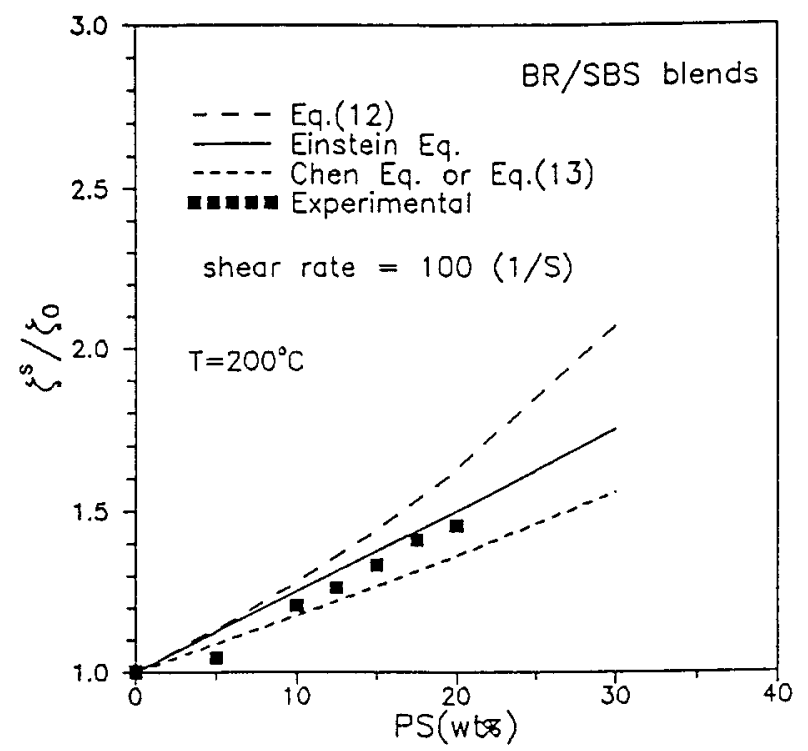

Figure 9 The relative viscosity predicted by eqs. (1), (8), (12), and (13) and experimental values measured at $200^{\circ} \mathrm{C}$ at shear rate of $100 \mathrm{~S}^{-1}$. 


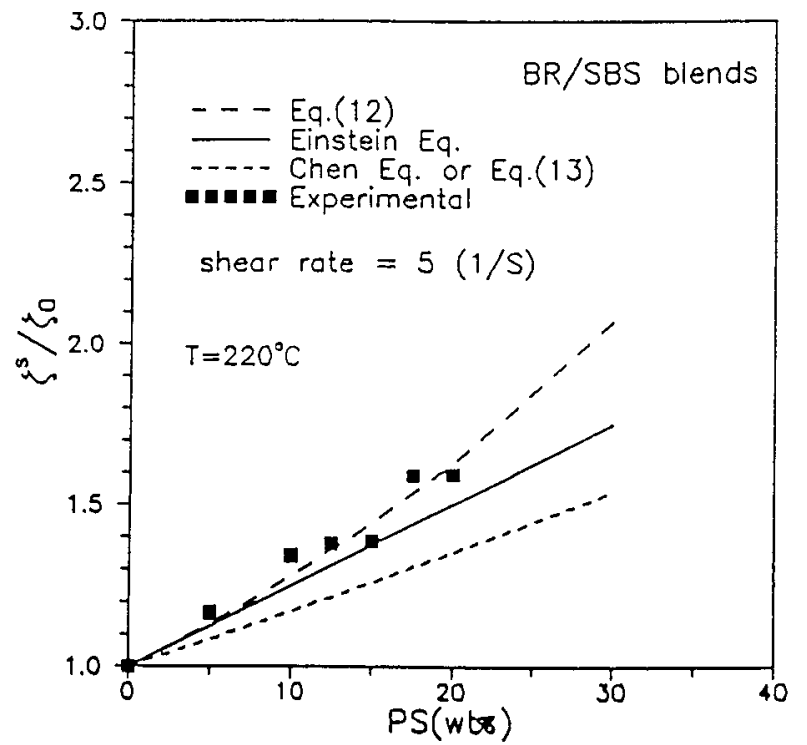

Figure 10 The relative viscosity predicted by eqs. (1), (8), (12), and (13) and experimental values measured at $220^{\circ} \mathrm{C}$ at shear rate of $5 \mathrm{~S}^{-1}$.

(8), eqs. (12) and (13), we must know the values of $a$ and $b$. The value of $a$ for pure BR at 200 and $220^{\circ} \mathrm{C}$ are determined as 0.8306 and 0.7975 , respectively, which is equivalent to $1-n$. The power law constant $\mathrm{n}$ can be obtained from curve fitting of the viscosity versus shear rate curve in Figures 4 and 5 into the power law equation [that is, eq. (2)] for pure BR. Also, we assume that the

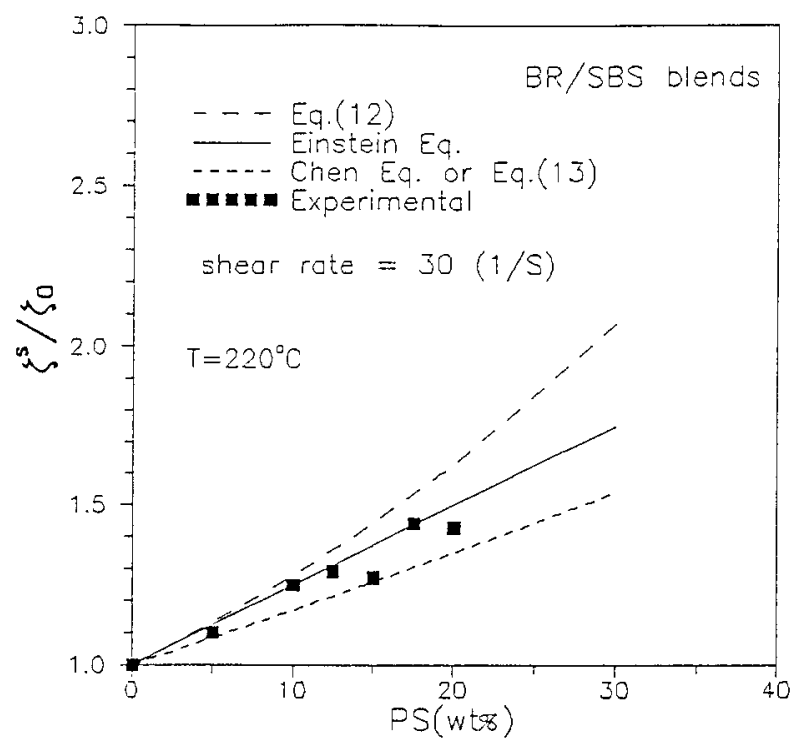

Figure 11 The relative viscosity predicted by eqs. (1), (8), (12), and (13) and experimental values measured at $220^{\circ} \mathrm{C}$ at shear rate of $30 \mathrm{~S}^{-1}$.

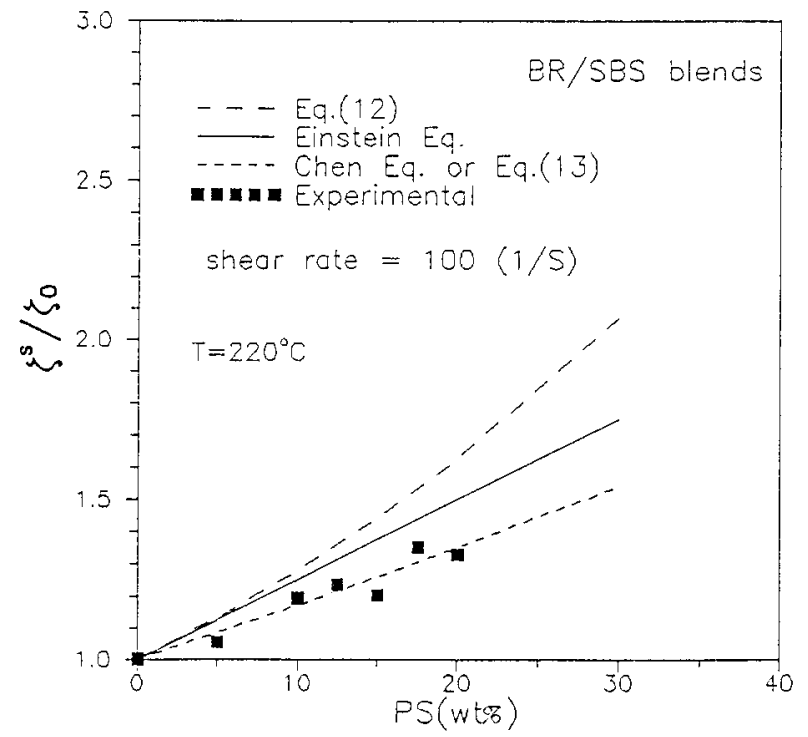

Figure 12 The relative viscosity predicted by eqs. (1), (8), (12), and (13) and experimental values measured at $220^{\circ} \mathrm{C}$ at shear rate of $100 \mathrm{~S}^{-1}$.

polymer blend fluid is an incompressible fluid suspension. Therefore, the value of $b$ can be obtained from eq. (6).

The predicted relative viscosities together with the experimental values measured at $200^{\circ} \mathrm{C}$ at shear rate of 5,30 , and $100 \mathrm{~S}^{-1}$ are shown in Figures 7-9, respectively. From these plots, we can find that the relative viscosity predicted by eq. (12) gives the better fit with the experimental

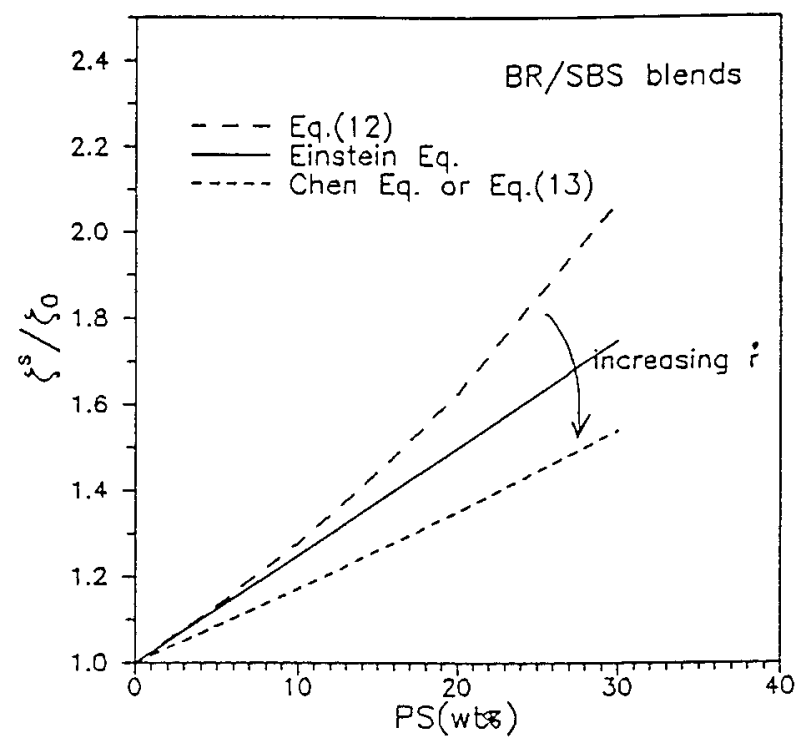

Figure 13 The comparison of theoretical data for eqs. (1), (8), (12), and (13). 
values at low shear rate, and eq. (13) [or eq. (8)] gives the better fit with the experimental values at high shear rate. A similar phenomenon can be seen in Figures $10-12$ at $220^{\circ} \mathrm{C}$.

The comparison of theoretical data for eqs. (1), (8), (12), and (13) are shown in Figure 13. It indicates that Einstein equation is close to our modified model at low $\dot{\gamma}$ [that is, eq. (12)] at a low content of the disperse phase. Chen's model is equivalent to our modified model at large $\dot{\gamma}$ [that is, eq. (13)]. It seems that eqs. (12) and (13) give the upper and lower bounds of the relative viscosity of the BR-SBS blends.

\section{CONCLUSION}

In this work, the morphology, $T_{g}$, and the viscosities of BR-SBS blends were investigated. From the morphology observation, the styrene component forms the discrete phase, and the butadiene component forms the continuous phase. From the differential scanning calorimetry analysis, it is seen that the presence of 2 glass transition temperatures indicates 2 phases, and the fact that the $T_{g}$ 's approach each other indicates that the BR-SBS blend is partial miscible. From the viscosity measurement, the viscosity of the BR-SBS blend increases with an increase in the content of PS. The behavior is in accord with the expected behavior of the filler effect. To predict the filler effect of PS phase in the BR-SBS blend, a modified model of Chen's is proposed to calculate the relative viscosity of the BR-SBS blends with different compositions. The agreement between the theoretical prediction and the experimental results is satisfactory.

\section{REFERENCES}

1. Chu L. H.; Guo S. H.; Chiu W. Y.; Tseng H. C. J. Appl. Polym. Sci. 1993, 49, 1791.

2. Turley S. G. J. Polym. Sci., Part C 1963, 1, 101.

3. Krause S. J. Macromol. Sci., Rev. Macromol. Chem. 1972, C7, 251.

4. Oene H. V.; Polymer Blends Vol. 1, Ch. 7, (Paul and Newman, Eds.), Academic Press, New York, 1978.

5. Einstein A. Ann. Phys. 1906, 19, 289.

6. Taylor G. I. Proc. R. Soc. 1932, A138, 41.

7. Nielsen L. E. Polymer Rheology Applied Science Publishers Ltd., London, UK, 1978.

8. Jeffery G. B. Proc. R. Soc. 1922, A102, 161.

9. Han C. D. Multiphase Flow in Polymer Processing, Academic Press, New York, (1987).

10. Simha R. J. Appl. Phys. 1952, 23, 1020.

11. Mooney M. J. Colloid Sci. 1952, 6, 162.

12. Chen C. H.; Cheng C. H. Mechanics of Materials 1997, p. 794 to appear.

13. Eshelby J. D. Proc. R. Soc. 1957, A241, 376.

14. Mori T.; Tanaka K. Acta Metall. 1973, 21, 571.

15. Aggarwal S. L.; Livigni R. A. Polym. Eng. Sci. 1977, $17,498$.

16. Wu S. Polym. Eng. Sci. 1987, 27, 335.

17. Olabisi O.; Robeson L. M.; Shaw M. T. PolymerPolymer Miscibility, Academic Press, New York, 1979. 